\title{
Supravalvular and Valvular Aortic Stenosis in Heterozygous Familial Hypercholesterolemia
}

\author{
Sima Rafeiyian Saeed Mojtahedzadeh Manouchehr Hekmat Nasim Naderi \\ Rezvan Nobahar Mohammad Jafar Hashemi Ali Kouhi \\ Cardiovascular Research Center, Shaheed Modarress Hospital, Shaheed Beheshti University of Medical Sciences, \\ Tehran, Iran
}

\section{Key Words}

Aortic stenosis · Supravalvular aortic stenosis · Familial hypercholesterolemia

\begin{abstract}
Objectives: To report calcified aortic stenosis due to hypercholesterolemia in two siblings. Case Presentation and Intervention: A 13-year-old boy with a history of dyspnea on exertion and a systolic murmur of aortic stenosis was referred to our center. Echocardiography showed combined valvular and supravalvular aortic stenoses with a good left ventricle systolic function and severe left ventricular hypertrophy. Two years later his 17-year-old sister was referred to the clinic with similar symptoms. Severe valvular aortic stenosis was detected by echocardiography. Selective coronary angiography showed significant involvement. The father had a history of hypercholesterolemia and confirmed coronary artery disease involving 3 vessels. Angiography showed anterioapical and inferiobasal hypokinesis with preserved left ventricle systolic function. The mother and the other two siblings did not have hyperlipidemia, thereby indicating heterozygous familial hypercholesterolemia in the two affected siblings. The siblings were managed with atrovastatin and nicotinic acid and cholestyramine was added stepwise. The father was treated with lovostatin. Conclusion: This report shows that severe hyperlipidemia in very young patients may be a risk factor for valvulopathy.
\end{abstract}

Copyright $\odot 2007$ S. Karger AG, Basel
(C) 2007 S. Karger AG, Basel

1011-7571/07/0164-0315\$23.50/0

Fax +4161306 1234

E-Mail karger@karger.ch

www.karger.com
Accessible online at: www.karger.com/mpp

\section{Introduction}

Calcific aortic stenosis is the commonest adult valvular heart condition and its prevalence has continued to rise, predominantly in older patients [1]. Although currently degenerative changes are the most important cause of valvular aortic stenosis [2], the latest development in pathophysiologic features of this valvulopathy help to find out evidences for the initiation and progression of this disease, similar to those described for atherosclerosis [3]. Lipid disturbances, in particular hypercholesterolemia, constitute an important factor in the initiation of valvular lesions and also in aortic orifice calcification [3]. Hyperlipidemia is associated with degenerative aortic valve stenosis, and the disease resembles the inflammatory process of atherosclerosis [4]. Studies have shown some relationship between cardiovascular risk factors and nonrheumatic severe calcific aortic stenosis $[3,4]$.

Certain preliminary clinical studies have shown that treatment with statins slows the progression of aortic stenosis [5], but the salutary mechanism of the statin may be cholesterol-lowering and/or anti-inflammatory $[5,6]$.

In this report, we describe two siblings with hypercholesterolemia with concomitant aortic stenosis. 
Table 1. Laboratory data of patients at the time of diagnosis

\begin{tabular}{lcccc}
\hline Parameter & $\begin{array}{l}\text { Brother } \\
(\mathrm{mg} / \mathrm{dl})\end{array}$ & $\begin{array}{l}\text { Normal range } \\
(\mathrm{mg} / \mathrm{dl})\end{array}$ & $\begin{array}{l}\text { Sister } \\
(\mathrm{mg} / \mathrm{dl})\end{array}$ & $\begin{array}{l}\text { Normal range } \\
(\mathrm{mg} / \mathrm{dl})\end{array}$ \\
\hline Total cholesterol & 347 & $130-204$ & 423 & $125-212$ \\
LDL cholesterol & 284 & $60-140$ & 356 & $60-150$ \\
HDL cholesterol & 38.2 & $30-65$ & 59.5 & $33-65$ \\
Triglyceride & 97 & $30-103$ & 74 & $32-122$ \\
FBS & 71 & $70-110$ & 84 & $70-110$ \\
Lipoprotein a & 35 & $0-60$ & 21 & $0-60$ \\
Apolipoprotein A1 & 71 & $115-190$ & 78 & $115-220$ \\
Apolipoprotein B & 151 & $17-160$ & 165 & $60-150$ \\
\hline
\end{tabular}

Normal range: sex related normal range. $\mathrm{LDL}=$ Low-density lipoprotein; HDL = high-density lipoprotein; FBS = fasting blood sugar.

\section{Case Reports}

\section{Case 1}

A 13-year-old boy with a history of dyspnea on exertion over several months and a systolic murmur of aortic stenosis (AS) on examination was referred to the pediatric heart clinic of Shaheed Modarress Hospital, affiliated to Shaheed Beheshti University of Medical Sciences, Tehran, Iran.

Echocardiography showed combined valvular and supravalvular AS, with a peak pressure gradient of $65 \mathrm{~mm} \mathrm{Hg}$ across the aortic valve, a good left ventricle systolic function and severe left ventricular hypertrophy. Cardiac catheterization showed $40 \mathrm{~mm} \mathrm{Hg}$ pressure for both gradients across the aortic valve and between aortic valve and ascending aorta. Ventriculography showed hypertrophied left ventricle, valvular and supravalvular AS and mild dilation of the aorta. Aortography confirmed valvular and supravalvular AS, but the coronary arteries were normal. Lipid profile showed cholesterolemia (table 1). The patient was scheduled for aortic valve replacement, but at surgery the aortic leaflets were normal and so only the supravalvular AS was corrected successfully, using a woven Dacron graft. Examination of specimen taken at surgery showed thickening of the valvular endocardium with extensive hyalinization and formation of a necrotic core including cholesterol clefts. Also, calcification was present in the peripheral part of the necrotic core. The patient was discharged without any complication and echocardiographies on follow-up were acceptable.

\section{Case 2}

Two years later, his 17-year-old sister was referred to our center due to dyspnea on exertion and effort angina with a New York Heart Association class of II. A loud midsystolic murmur with a grade of IV/VI was heard at the right second intercostal space. Severe valvular AS with a peak gradient of $154 \mathrm{~mm} \mathrm{Hg}$ was detected by echocardiography. The patient had severe concentric left ventricular hypertrophy, and the aortic valve appeared severely thickened and calcified. Physical examination revealed that she had xanthelasma on her eyelids and xanthoma tendinosum on the elbows. Plasma lipid profile showed hyperlipidemia (table 1). Cardiac catheterization showed a pressure gradient of $140 \mathrm{~mm} \mathrm{Hg}$ across the aortic valve, and aortography demonstrated hypoplastic valsalva sinuses and severe calcification of the aortic valve. Selective coro- nary angiography showed intimal irregularity in the left anterior descending artery and significant stenosis of the diagonal and obtuse marginal branches; the right coronary system was normal. Due to hypoplastic aortic root Konno-Rastan procedure was performed successfully and postoperation echocardiography indicated acceptable hemodynamic parameters. Examination of tissue samples showed distorted aortic leaflets with extensive hyaline fibrosis, mural endocardium, revealing fibrous thickening. Hypertrophic myocytes with atypical nuclei were seen.

In both cases the hyperlipidemia was managed with atorvastatin and nicotinic acid, and cholestyramine was added stepwise. However, because of incomplete response and high lipid levels, plasmapheresis was recommended, but both patients refused to undergo plasmapheresis. Because of high total and low-density lipoprotein (LDL) cholesterol in these siblings, the rest of the family was advised to check their serum lipid levels. The mother and two other children had normal cholesterol levels, but the 46-yearold father had a history of hypercholesterolemia for many years, and he also had a confirmed history of coronary artery disease involving 3 vessels. He was asymptomatic until 4 years ago, when angiography showed anteroapical and inferobasal hypokinesia with preserved left ventricle systolic function (ejection fraction = $50 \%)$. The left anterior descending artery was $90 \%$ segmentally stenotic after D1 (till D3). D1, D2 and D3 were $90 \%$ stenotic, proximally. The left circumflex artery was $70 \%$ stenotic proximally and $99 \%$ stenotic after the second obtuse marginal branch. The right coronary artery was totally cut off with fair runoff proximally. He did not undergo revascularization procedures, is under treatment with lovastatin and till now is asymptomatic. He does not have valvular cardiac involvement.

\section{Discussion}

AS is the most commonly encountered valvular heart disease in the elderly, with approximately $2-3 \%$ of individuals over 65 years of age afflicted [1]. The prevalence is continuing to rise, with predominance in older patients 
who frequently undergo successful aortic valve replacement [1]. The most common cause of AS is slow progressive calcific degeneration, with an asymptomatic period which can last decades [3]; therefore, it was thought that calcific AS resulted from aging and 'wear and tear' of the aortic valve [7], hence considered as degenerative or senile-type condition. Currently, this perception has been changing, and understanding the risk factors of AS and its histological characteristics has led to new insights into how it develops [3]. Some similarities have been found between the lesions of AS and atheromatous coronary artery disease [3] and calcific AS is being increasingly recognized as an inflammatory, atheromatous and potentially modifiable disease. The young age of the two siblings indicates etiologic factors other than wear and tear due to ageing processes.

In view of the extremely high total and LDL cholesterol levels, normal triglyceride and near normal apolipoprotein levels (table 1), premature coronary lesions and probable autosomal dominant inheritance (as father, brother and sister are involved), familial hypercholesterolemia would be the most probable type of hyperlipidemia in this family. Although cutaneous xanthomas and aortic valve/root involvement are rarely seen in heterozygotes and typically present with cardiovascular disease in the 30s and 40s, and since the mother and two other siblings are not hyperlipidemic, our two patients probably are heterozygous. Supravalvular AS is also seen in some genetic diseases such as familial supravalvular AS, a rare (1 in 20,000 live births) dominant condition, and Williams-Beuren syndrome [8]. These two are presumed to be due to a quantitative deficiency of elastin rather than a qualitative defect caused by a mutation in the elastin gene located at 7q11-23. WilliamsBeuren syndrome can be distinguished from familial supravalvular AS by the characteristic dysmorphic appearances and mental retardation [8]. In familial supravalvular
AS the defining feature of the malformation is aortic narrowing at the level of the sinotubular junction, but in some cases there is a narrowing of the entire ascending aorta and arch branches [9]. In addition to the aorta, other major arteries, including the pulmonary, carotid, cerebral, renal and coronary arteries, may also be involved [9].

The diagnosis of Williams syndrome could be ruled out in the two siblings, since they did not have dysmorphic appearance and had good mental development. Also, there could not be supravalvular AS in situ because they had mainly localized pathology in the aorta and not other vessels, and aortic leaflets alone were involved. Furthermore, their father had no valvular involvement. However, the most accurate method for diagnosing of familial supravalvular AS is to test for the presence of elastin gene, which unfortunately was not performed in our patients.

Familial hypercholesterolemia, with a heterozygous disease incidence of 1 in 500, is the most common known monogenic form of inherited metabolic disease. It is characterized by an elevation of LDL cholesterol concentration in plasma as in our two patients, leading to deposition of excess LDL-derived cholesterol in tendons, skin and arteries. Heterozygous familial hypercholesterolemia patients usually have a twofold increase in total cholesterol and LDL-derived cholesterol and typically present with cardiovascular disease in their 30 s and 40 s, although our patients were teenagers.

\section{Conclusion}

Hypercholesterolemia constitutes an important factor in the initiation of valvular lesions, even at a young age. Thus, hyperlipidemia, when seen in young patients, should be considered a risk factor and screening for lipid disturbances in aortic valvulopathy is recommended.

\section{References}

1 Cowell SJ, Newby DE, Boon NA, Elder AT: Calcific aortic stenosis: same old story? Age Ageing 2004;33:538-544.

$\checkmark 2$ Podolec P, Kopec G, Rubis P, Kablak-Ziembicka A, Rokita E, Tracz W: Calcific and degenerative aortic stenosis - pathogenesis and new possibilities of treatment. Przegl Lek 2004;61:604-608.
3 Weyman AE: Exploring the relationship between hyperlipidemia and aortic stenosis. Rev Cardiovasc Med 2002;3:160-161.

4 Rossebo AB, Pedersen TR: Hyperlipidaemia and aortic valve disease. Curr Opin Lipidol 2004;15:447-451.

5 Quinn DW Jr, Spinler SA: Efficacy of statins in preventing progression of aortic stenosis. Am J Health Syst Pharm 2005;62:979-981.

6 Pate GE, Tahir MN, Murphy RT, Foley JB: Anti-inflammatory effects of statins in patients with aortic stenosis. J Cardiovasc Pharmacol Ther 2003;8:201-206.
Calcific Aortic Stenosis in

Hypercholesterolemia
Pomerance A: Ageing changes in human heart valve. Br Heart J 1967;29:222-231.

$\checkmark 8$ Keating MT: Genetic approaches to cardiovascular disease: supravalvular aortic stenosis, Williams syndrome, and long-QT syndrome. Circulation 1995;92:142-147.

-9 Milewicz DM, Urban Z, Boyd C: Genetic disorders of the elastic fiber system. Matrix Biol 2000;19:471-480. 